\title{
EXTERNAL ANALYSIS OF BOUNDARY POINTS OF CONVEX SETS: ILLUMINATION AND VISIBILITY
}

\author{
JOSÉ PEDRO MORENO and ALBERTO SEEGER
}

\begin{abstract}
The purpose of this work is studying the geometry of the boundary $\partial K$ of a solid closed convex set $K$ in a normed space. In a recent paper of ours, such a study has been carried out with the help of supporting cones and drops. Now, illuminated sets and visible sets are the main tools of analysis.
\end{abstract}

\section{Introduction}

This is the second and last part of a work initiated in [14]. It concerns a central issue of classical and modern convex analysis, namely, the description of some geometric properties of boundary points of convex sets. To be more precise, we are interested in examining the boundary $\partial K$ of a given element $K$ taken from the class

$$
\Xi(X) \equiv \text { solid closed convex proper subsets of } X .
$$

Here, $(X,\|\cdot\|)$ stands for a real normed space of dimension greater than or equal to two. As usual, a proper subset of $X$ is any set different from the whole space $X$. That $K$ is solid simply means that its topological interior is nonempty.

Strict convexity and local uniform rotundity are two among other properties of boundary points that we are interested in. The notion of local uniform rotundity is one of the main topics in renorming theory, a branch of functional analysis devoted to find equivalent norms with good geometrical properties. Local uniform rotundity has been traditionally considered only as a matter for norms and, consequently, closed balls. However, the definition can be easily generalized to arbitrary solid closed convex sets.

Our methodology consists in introducing a few multivalued maps of the form $F_{K}: X \rightrightarrows X$ and see what happens with the set $F_{K}(x)$ when the argument $x \in X$ approaches the boundary of $K$ from the exterior.

Received April 11, 2008. 
The main protagonist in [14] was the multivalued map $T_{K}: X \rightrightarrows X$ given by

$$
T_{K}(x)=\operatorname{cl}\left[\bigcup_{\alpha>0} \alpha(K-x)\right],
$$

where "cl" stands for closure. One refers to (1) as the supporting cone to $K$ at $x$ (cf. [18]). For us, a point $x \in X$ of interest is not one lying in the set $K$, but in its exterior. We are then deviating from the common practice of convex analysis and adopting a rather unorthodox approach. A secondary role way played by closed drops, which are sets of the form

$$
D_{K}(x)=\operatorname{cl}(x+[0,1](K-x)) .
$$

Such sets are extensively used in the geometric analysis of Banach spaces (cf. [9], [13], [17]).

In the present paper we join the community of those who are interested in illumination and visibility of convex sets. As main tool of analysis, we consider the sets

$$
\begin{aligned}
L_{K}(x) & =\{u \in X: u \text { is illuminated by } x\}, \\
V_{K}(x) & =\{u \in X: u \text { is visible from } x\}, \\
W_{K}(x) & =\{u \in X: u \text { is weakly visible from } x\},
\end{aligned}
$$

as functions of the parameter $x \in X$.

The precise definition of the above sets is recalled next. For an easy geometric understanding of Definition 1.1, it is helpful to see the convex set $K$ as a sort of dark body whose boundary needs to be illuminated, and the point $x$ as a source of light that is placed somewhere in the exterior. We are not concerned here with the illumination of a nonconvex body by means of a source of light located in the interior. Internal illumination is an entirely different story. Following a common practice in topology, the interior and the exterior of $K$ are denoted by $\operatorname{int}(K)$ and $\operatorname{ext}(K)$, respectively.

Definition 1.1. Given a set $K \in \Xi(X)$ and points $x \in \operatorname{ext}(K)$ and $u \in \partial K$, one says that:

(i) $u$ is illuminated by $x$ if the ray $\{x+\lambda(u-x): \lambda \geq 1\}$ meets int $(K)$.

(ii) $u$ is visible from $x$ if the line segment $[x, u]$ intersects $K$ only in $u$.

(iii) $u$ is weakly visible from $x$ if $[x, u]$ does not meet int $(K)$.

According to a survey paper by Martini and Soltan [10], the concept of illumination stated in Definition 1.1 goes back to Hadwiger [8]. An appropriate reference for the concept of visibility is Valentine [19]. Under a slightly 
different name, the notion of weak visibility is considered for the first time by Buchman and Valentine [5].

As expected, by letting $x$ move in the exterior of $K$, one recovers useful information on the structure of $\partial K$. A particularly striking result in this sense is Theorem 3.10: locally uniformly rotund points in $\partial K$ are fully characterized by the behavior of the multivalued map $V_{K}$.

\section{Illumination}

By construction, the multivalued maps $L_{K}, V_{K}, W_{K}: X \rightrightarrows X$ are emptyvalued when evaluated at a point in $K$, and nonempty-valued when evaluated at a point in $\operatorname{ext}(K)$. These maps are related to each other through the inclusions

$$
L_{K}(x) \subset V_{K}(x) \subset W_{K}(x) .
$$

More often than not, these inclusions are strict. In Figure 1 the situation is as follows: $v$ is illuminated by $x$; the point $u$ is visible, but not illuminated; the point $w$ is weakly visible, but not visible.

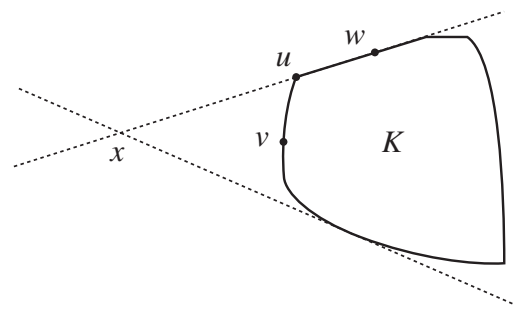

FIGURE 1. The boundary points $v, u$ and $w$ have a different status.

Among other things, we shall discuss the semicontinuity behavior of each one of these maps. Recall that a multivalued map $\Gamma: X \rightrightarrows X$ on a normed space is said to be lower-semicontinuous at a reference point $\bar{x} \in X$ if

$$
\Gamma(\bar{x}) \subset \liminf _{x \rightarrow \bar{x}} \Gamma(x) .
$$

The natural counterpart of lower-semicontinuity is a concept called outersemicontinuity. One says that $\Gamma$ is outer-semicontinuous at $\bar{x} \in X$ if

$$
\limsup _{x \rightarrow \bar{x}} \Gamma(x) \subset \Gamma(\bar{x}) .
$$

The upper and lower limits mentioned above are understood in the Painlevé- 
Kuratowski sense, i.e.,

$$
\begin{aligned}
\limsup _{x \rightarrow \bar{x}} \Gamma(x) & =\left\{y \in X: \liminf _{x \rightarrow \bar{x}} \operatorname{dist}[y, \Gamma(x)]=0\right\}, \\
\liminf _{x \rightarrow \bar{x}} \Gamma(x) & =\left\{y \in X: \lim _{x \rightarrow \bar{x}} \operatorname{dist}[y, \Gamma(x)]=0\right\} .
\end{aligned}
$$

Clearly, the illumination map $L_{K}$ is not outer-semicontinuous. However, one has:

Proposition 2.1. If $K \in \Xi(X)$, then $L_{K}: X \rightrightarrows X$ is lower-semicontinuous.

Proof. As a preliminary step, let us obtain an explicit formula for the inverse $L_{K}^{-1}: X \rightrightarrows X$ of the map $L_{K}$. By definition, one has $L_{K}^{-1}(u)=\{x \in$ $\left.X: u \in L_{K}(x)\right\}$. Of course, $L_{K}^{-1}(u)=\emptyset$ in case $u \notin \partial K$. We claim that

$$
L_{K}^{-1}(u)=u-\operatorname{int}\left[T_{K}(u)\right]
$$

for all $u \in \partial K$. Both sides of (3) are contained in the exterior of $K$. Take $u \in \partial K$ and $x \in \operatorname{ext}(K)$. In view of [14, Lemma 2.1], we need to prove that

$$
u \in L_{K}(x) \Longleftrightarrow x \in u-E_{\operatorname{int}(K)}(u)
$$

with $E_{C}(u)=\bigcup_{\alpha>0} \alpha(C-u)$. If $u \in L_{K}(x)$, then $z_{\lambda}=x+\lambda(u-x) \in \operatorname{int}(K)$ for some $\lambda>1$. Hence,

$$
-(x-u)=(\lambda-1)^{-1}\left(z_{\lambda}-u\right) \in E_{\operatorname{int}(K)}(u) .
$$

Conversely, suppose true the right-hand side of (4). Then, $x=u-\alpha(z-u)$ with $\alpha>0$ and $z \in \operatorname{int}(K)$. In such a case, $u \in L_{K}(x)$ because

$$
z=\left(\frac{-1}{\alpha}\right) x+\left(1+\frac{1}{\alpha}\right) u=x+\lambda(u-x) \in \operatorname{int}(K)
$$

with $\lambda=1+(1 / \alpha)>1$. The proof of formula (3) is thus complete. Note that the set on the right-hand side of (3) is open. This observation leads straightforwardly to the lower-semicontinuity of $L_{K}$. Indeed, a multivalued map whose inverse has open values is necessarily lower-semicontinuous.

REMARK 2.2. Formula (3) is a result of interest by its own. It tells in which region of $\operatorname{ext}(K)$ one must place the source of light $x$ if one wishes the boundary point $u$ to be illuminated. Parenthetically, we mention that a famous problem of illumination theory is that of determining the minimal number $p$ of points $x_{1}, \ldots, x_{p}$ in $\operatorname{ext}(K)$ that are needed to have the full boundary illuminated, i.e.,

$$
\partial K=L_{K}\left(x_{1}\right) \cup \ldots \cup L_{K}\left(x_{p}\right) .
$$


The answer to this difficult question depends on whether $K$ is a polytope or a smooth convex body. The reader interested in this question can find a wealth of information in the work by Bezdek [2], [3], or in the survey paper by Martini and Soltan [10].

Further properties of illuminated sets are stated in the next proposition. The literature on illumination is quite vast and the terminology employed by different authors in not always the same. Probably some portions of Proposition 2.3 are already known.

Proposition 2.3. Let $K \in \Xi(X)$. For any $x \in \operatorname{ext}(K)$, one has:

(a) $L_{K}(x)$ is arc-connected.

(b) $L_{K}(x)$ contains a set which is homeomorphic to the interval $[0,1]$. In particular, $L_{K}(x)$ is uncountable.

(c) $L_{K}(x)$ is open as subset of $\partial K$. In fact, $L_{K}(x)=O_{K}(x) \cap \partial K$ with

$$
O_{K}(x)=x+\bigcup_{t \in] 0,1[}(\operatorname{int}(K)-x)=\operatorname{int}\left[D_{K}(x)\right] .
$$

Proof. For manifold reasons, it is useful to represent $L_{K}(x)$ as the image of an open convex set under a continuous function. Indeed, one has the representation formula

$$
L_{K}(x)=\left\{\Phi_{x}(z): z \in \operatorname{int}(K)\right\},
$$

where $\Phi_{x}: \operatorname{int}(K) \rightarrow \partial K$ is defined by

$$
\Phi_{x}(z) \equiv \text { unique element in }[x, z] \cap \partial K .
$$

To see that $\Phi_{x}$ is continuous, consider a reference point $z \in \operatorname{int}(K)$ and a sequence $\left\{z_{n}\right\}_{n \in \mathrm{N}} \rightarrow z$. For each $n \in \mathrm{N}$, there is a unique $\left.t_{n} \in\right] 0,1[$ such that

$$
\Phi_{x}\left(z_{n}\right)=x+t_{n}\left(z_{n}-x\right) .
$$

We claim that $\left\{t_{n}\right\}_{n \in \mathrm{N}}$ converges to the unique solution $\left.t \in\right] 0,1[$ of the equation

$$
\Phi_{x}(z)=x+t(z-x) .
$$

By a compactness argument, $\left\{t_{n}\right\}_{n \in \mathrm{N}}$ admits a cluster point $\bar{t}$ in [0,1]. In view of (7), it follows that $\bar{u}=x+\bar{t}(z-x)$ is a cluster point of the sequence $\left\{\Phi_{x}\left(z_{n}\right)\right\}_{n \in \mathrm{N}}$. The closedness of $\partial K$ ensures that $\bar{u} \in \partial K$. But the segment $[x, z]$ meets $\partial K$ only at $\Phi_{x}(z)$. Hence, $\bar{u}=\Phi_{x}(z)$ and $\bar{t}=t$. This proves our claim and confirms the continuity of $\Phi_{x}$. The representation formula (6) has 
several consequences. First of all, it implies the arc-connectedness of $L_{K}(x)$. Indeed, if $u_{0}, u_{1} \in L_{K}(x)$, then one can find $z_{0}, z_{1} \in \operatorname{int}(K)$ such that $u_{0}=$ $\Phi_{x}\left(z_{0}\right)$ and $u_{1}=\Phi_{x}\left(z_{1}\right)$. Since

$$
t \in[0,1] \mapsto z_{t}=(1-t) z_{0}+t z_{1} \in \operatorname{int}(K)
$$

is a continuous arc joining $z_{0}$ and $z_{1}$, it follows that

$$
t \in[0,1] \mapsto \Phi_{x}\left(z_{t}\right) \in L_{K}(x)
$$

is a continuous arc joining $u_{0}$ and $u_{1}$. This takes care of (a). On the other hand, if one chooses $z_{0}, z_{1} \in \operatorname{int}(K)$ so that $\left\{x, z_{0}, z_{1}\right\}$ are not on the same line, then $u_{0}=\Phi_{x}\left(z_{0}\right)$ and $u_{1}=\Phi_{x}\left(z_{1}\right)$ are two different points in $L_{K}(x)$. Observe that $L_{K}(x)$ contains not just $u_{0}$ and $u_{1}$, but also the whole arc $\left\{\Phi_{x}\left(z_{t}\right): t \in[0,1]\right\}$. The latter set is homeomorphic to $[0,1]$ because $(8)$ is continuous and injective. This proves (b).

The first equality in (5) is the definition of $O_{K}(x)$. Clearly, $O_{K}(x)$ is open in $X$. Although one could dispense with the second equality in (5), we prefer to provide a geometric interpretation for the set $O_{K}(x)$. That $O_{K}(x)$ is the interior of the closed drop $D_{K}(x)$ can be proven by proceeding as in [14, Lemma 2.1]. Finally, observe that

$$
\begin{aligned}
u \in O_{K}(x) \cap \partial K & \Longleftrightarrow u=x+t(z-x) \in \partial K \text { with }(t, z) \in] 0,1[\times \operatorname{int}(K) \\
& \Longleftrightarrow u=\Phi_{x}(z) \text { with } z \in \operatorname{int}(K) .
\end{aligned}
$$

In view of (6), the last condition amounts to saying that $u \in L_{K}(x)$.

The next result is an extension of Proposition 2.3(b). Roughly speaking, Proposition 2.4 says the following: if $K$ lives in the Euclidean space $\mathrm{R}^{d}$, then $L_{K}(x)$ contains a surface of dimension $d-1$; in the infinite dimensional case, $L_{K}(x)$ contains a surface whose dimension can be taken arbitrarily large. We view a $p$-dimensional surface as a set which is homeomorphic to the $p$ dimensional simplex

$$
\Lambda_{p}=\left\{\lambda \in \mathrm{R}_{+}^{p+1}: \sum_{i=0}^{p} \lambda_{i}=1\right\} .
$$

So, our concept of "surface" is purely topological and leaves aside all reference to differentiability.

Proposition 2.4. Let $p$ be any positive integer smaller than $\operatorname{dim} X$. If $x$ lies in the exterior of $K \in \Xi(X)$, then $L_{K}(x)$ contains a set which is homeomorphic to the p-dimensional simplex. 
Proof. Given that $p$ is smaller than $\operatorname{dim} X$, it is possible to find points $z_{0}, z_{1}, \ldots, z_{p}$ in the interior of $K$ such that $\left\{z_{0}-x, z_{1}-x, \ldots, z_{p}-x\right\}$ form a collection of linearly independent vectors. For each $\lambda \in \Lambda_{p}$, the point $z_{\lambda}=\sum_{i=0}^{p} \lambda_{i} z_{i}$ belongs to $\operatorname{int}(K)$, and therefore

$$
H(\lambda)=\Phi_{x}\left(z_{\lambda}\right)
$$

belongs to $L_{K}(x)$. We claim that $H: \Lambda_{p} \rightarrow X$ is an injection. Let $\lambda, \mu \in \Lambda_{p}$ be such that $H(\lambda)=H(\mu)$. Hence, $\left\{x, z_{\lambda}, z_{\mu}\right\}$ are on the same line. In other words,

$$
z_{\mu}-x=\alpha\left(z_{\lambda}-x\right)
$$

for some $\alpha>0$. After a short rearrangement, one gets

$$
\sum_{i=0}^{p}\left(\mu_{i}-\alpha \lambda_{i}\right)\left(z_{i}-x\right)=0 .
$$

By linear independence, one has $\mu_{i}=\alpha \lambda_{i}$ for all $i \in\{0,1, \ldots, p\}$. Summing up, one gets $\alpha=1$, and then $\mu=\lambda$. Thus, $H$ acts injectively on $\Lambda_{p}$. Equivalently, $H$ is a bijection between $\Lambda_{p}$ and $S=H\left(\Lambda_{p}\right)$. The function $H: \Lambda_{p} \rightarrow S$ is continuous because it is a composition of continuous functions. A standard topological argument yields then the compactness of $S$ and the continuity of the inverse function $H^{-1}: S \rightarrow \Lambda_{p}$. Summarizing, we have shown that $L_{K}(x)$ contains $S$, a set which is homeomorphic to $\Lambda_{p}$.

\section{From illumination to visibility}

We now shift the attention from illumination to visibility. The proposition below shows that there is a connection between both concepts.

Proposition 3.1. Let $K \in \Xi(X)$. For any $x \in \operatorname{ext}(K)$, one has:

(a) $V_{K}(x)$ is arc-connected.

(b) $V_{K}(x) \subset \operatorname{cl}\left[L_{K}(x)\right]$.

Proof. Let $u_{0}, u_{1} \in V_{K}(x)$. If both points are in $L_{K}(x)$, then one can join them by means of a continuous arc as in Proposition 2.3(a). Suppose, for instance, that $u_{1} \notin L_{K}(x)$. Pick any $\hat{z} \in \operatorname{int}(K)$ and consider the curve

$$
t \in\left[0,1\left[\mapsto \gamma(t)=\Phi_{x}\left(\hat{z}+t\left(u_{1}-\hat{z}\right)\right) \in L_{K}(x) \subset V_{K}(x)\right.\right.
$$

emanating from $\hat{u}=\Phi_{x}(\hat{z})$. As we saw already in the proof of Proposition 2.3, the function $\gamma$ is continuous. Since one wishes to arrive at the point $u_{1}$, one extends $\gamma$ to the closed interval $[0,1]$ by setting $\gamma(1)=u_{1}$. We claim that 
such an extension is continuous at $t=1$. To see this, consider any sequence $\left\{t_{n}\right\}_{n \in \mathrm{N}}$ in $\left[0,1\right.$ [ converging to 1 . The corresponding sequence $\left\{\gamma\left(t_{n}\right)\right\}_{n \in \mathrm{N}}$ lies in the triangle $\Delta=\operatorname{co}\left\{x, u_{1}, \hat{z}\right\}$, and therefore it has a cluster point $\bar{u} \in \Delta$. Necessarily $\bar{u} \in\left[x, u_{1}\right]$, by the definition of $\gamma$ over [0, 1[. Now, if $\bar{u}$ were not equal to $u_{1}$, then $u_{1}$ would not be visible from $x$, hence $\bar{u}=u_{1}$. This confirms that $\gamma:[0,1] \rightarrow V_{K}(x)$ is a continuous arc joining $\Phi_{x}(\hat{z})$ and $u_{1}$. In the same way, one constructs another continuous arc joining $\Phi_{x}(\hat{z})$ and $u_{0}$, and then one combines both pieces. Part (b) is implicit in the proof technique of part (a).

Corollary 3.2. If $K \in \Xi(X)$, then $V_{K}: X \rightrightarrows X$ is lower-semicontinuous.

Proof. It is a matter of combining the leftmost inclusion in (2), Proposition 3.1(b), and Proposition 2.1.

That illumination and visibility are two related concepts is also clear from the theorem of Bogopolskii and Vasiliev [4]. We are quoting this result from the survey paper [10], in where all mathematical statements are given in a finite dimensional context. We have not seen the proof given in the original Russian source, but we did check that Theorem 3.3 holds true in an infinite dimensional setting as well. We shall not write here our own proof for avoiding possible repetitions with the existing literature.

Theorem 3.3. Let $K \in \Xi(X), x \in \operatorname{ext}(K)$, and $u \in \partial K$. Then, $u$ is illuminated by $x$ if and only if there is a neighborhood $N$ of $u$ such that each point in $N \cap \partial K$ is visible from $x$.

Formulated in a shorter but less readable manner, Theorem 3.3 says that

$$
L_{K}(x)=\operatorname{int}_{\partial K}\left[V_{K}(x)\right]
$$

for every $x \in \operatorname{ext}(K)$. Here, the symbol int $_{\partial K}$ indicates the interior relative to the topology of $\partial K$. Formula (9) corroborates the fact that $L_{K}(x)$ is open as subset of $\partial K$ (cf. Proposition 2.3).

We continue the presentation of visibility with a result that is specific to the two dimensional case. That Proposition 3.4 is not true in higher dimensions will be illustrated with the help of an example.

Proposition 3.4. Let $K$ be a solid closed convex set in $\mathrm{R}^{2}$. Then, $V_{K}(x)$ is closed for all $x \in \operatorname{ext}(K)$.

Proof. Let $x \in \operatorname{ext}(K)$. Let $\left\{u_{n}\right\}_{n \in \mathrm{N}}$ be a sequence in $V_{K}(x)$ converging to a certain $u$. We must prove that $u \in V_{K}(x)$. Since $\partial K$ is closed, one knows already that $u \in \partial K$. Ab absurdo, suppose that $u \in \partial K \backslash V_{K}(x)$. Hence, there exists a point $w \in K$ such that $w \in[x, u]$ and $w \neq u$. Consider the line $L$ which contains $x$ and $u$. The set $\mathrm{R}^{2} \backslash L$ is divided in two open half-planes, say 
$P_{1}$ and $P_{2}$. There are two possibilities for consideration: either int $(K)$ meets only one half-plane, say $P_{1}$, or int $(K)$ meets both half-planes. In the first case, pick $z \in P_{1} \cap \operatorname{int}(K)$ and a small $\varepsilon>0$ such that

$$
B(u, \varepsilon) \cap K \subset x+E_{[w, z]}(x),
$$

where $B(u, \varepsilon)$ stands for the closed ball of radius $\varepsilon$ centered at $u$, and

$$
E_{[w, z]}(x)=\{\alpha(y-x): \alpha>0, y \in[w, z]\} .
$$

We now examine what happens inside the set $x+E_{[w, z]}(x)$. Observe that $V_{K}(x) \cap\left(x+E_{[w, z]}(x)\right)$ is a subset of the triangle $\Delta=\operatorname{co}\{x, w, z\}$. Since $u \notin \Delta$, one can choose $\varepsilon^{\prime}<\varepsilon$ such that $B\left(u, \varepsilon^{\prime}\right) \cap \Delta=\emptyset$, hence $B\left(u, \varepsilon^{\prime}\right) \cap$ $V_{K}(x)=\emptyset$. This contradicts that $u_{n} \in B\left(u, \varepsilon^{\prime}\right) \cap V_{K}(x)$ for $n$ large enough. The case in which $\operatorname{int}(K)$ meets both half-planes can be proved in an analogous way.

ExAmple 3.5. In the usual three dimensional Euclidean space, let $K$ be the smallest convex set containing the point $(0,0,1)$ and the circle $C=\{z \in$ $\left.\mathrm{R}^{3}:\left(z_{1}-1\right)^{2}+z_{2}^{2}=1, z_{3}=0\right\}$. It is easy to see that $x=(0,0,2)$ is in the exterior of $K$. With the exception of $(0,0,0)$, all other points of $C$ belong to the set $V_{K}(x)$. This implies that $V_{K}(x)$ is not closed.

Remark 3.6. By the way, Example 3.5 shows that the inclusion in Proposition 3.1(b) can be strict when the dimension of $X$ is higher than 2. Indeed, $\operatorname{cl}\left[L_{K}(x)\right]$ is always closed, but $V_{K}(x)$ need not be closed.

It comes without surprise that $V_{K}$ has closed values if the set $K$ is polyhedral. In fact, polyhedrality takes care not just of closedness, but it adds more structure to the set $V_{K}(x)$.

Proposition 3.7. Let $K$ be a solid convex polyhedral set in the Euclidean space $\mathrm{R}^{d}$, and let $x \in \operatorname{ext}(K)$. Then, $V_{K}(x)$ is a union of facets of $K$.

Proof. By polyhedrality, $K$ is expressible as intersection

$$
K=\bigcap_{i=1}^{m}\left\{z \in \mathrm{R}^{d}: f_{i}(z) \leq \beta_{i}\right\}
$$

of finitely many affine half-spaces. Here, $f_{i}: \mathrm{R}^{d} \rightarrow \mathrm{R}$ is a nonzero linear function and $\beta_{i}$ is a scalar. There is no loss of generality in assuming that $f_{i}$ is a norm-one linear function, i.e.,

$$
\sup _{\|\xi\| \leq 1} f_{i}(\xi)=1
$$


One may also assume that (10) is a minimal representation of $K$ in the sense that

$$
F_{i}=K \cap f_{i}^{-1}\left(\left\{\beta_{i}\right\}\right)=\left\{z \in K: f_{i}(z)=\beta_{i}\right\}
$$

is a facet of $K$, i.e., a face of $K$ of dimension $d-1$. We claim that $V_{K}(x)$ is expressible in the form

$$
V_{K}(x)=\bigcup_{i \in I(x)} F_{i}
$$

with $I(x)=\left\{i \in\{1, \ldots, m\}: f_{i}(x)>\beta_{i}\right\}$. Since $x \in \operatorname{ext}(K)$, the index set $I(x)$ is nonempty. Suppose that $u \in F_{i}$ for some $i \in I(x)$. Hence, for all $t \in[0,1[$, one has

$$
f_{i}((1-t) x+t u)=(1-t) f_{i}(x)+t f_{i}(u)>\beta_{i},
$$

i.e., the segment $\left[x, u\left[\right.\right.$ lies in the exterior of $K$. This shows that $u \in V_{K}(x)$. Conversely, suppose that $u \in V_{K}(x)$. Let $J$ be the set of all indices $i \in$ $\{1, \ldots, m\}$ such that $f_{i}(u)=\beta_{i}$. The set $J$ contains at least one element because $u \in \partial K$. It is not difficult to see that

$$
\left.\left.B(u, \varepsilon) \cap\left[\bigcap_{i \in J} f_{i}^{-1}(]-\infty, \beta_{i}\right]\right)\right] \subset K
$$

for some $\varepsilon>0$ small enough. Indeed, given the normalization condition (11) and the fact that $f_{i}(u)<\beta_{i}$ for all $i \notin J$, one can take

$$
\varepsilon<\min _{i \notin J}\left\{\beta_{i}-f_{i}(u)\right\} .
$$

Now, if we had $f_{i}(x) \leq \beta_{i}$ for all $i \in J$, then the inclusion (13) would imply that

$$
w=u+\varepsilon(x-u)\|x-u\|^{-1} \in K,
$$

contradicting that $u$ is visible from $x$. Hence, $I(x) \cap J \neq \emptyset$. This proves the existence of an index $i \in I(x)$ such that $u \in F_{i}$.

Formula (12) is something more precise than the statement of Proposition 3.7, but it requires having the representation (10) of $K$ at hand.

\subsection{Characterizing local uniform rotundity via visibility}

The next theorem shows how Valentine's concept of visibility has a bearing in the problem of detecting locally uniformly rotund points in the boundary of a given set. We state first a definition and a lemma. 
Definition 3.8. A point $u$ in the boundary of $K \in \Xi(X)$ is called locally uniformly rotund (LUR, in short) if $\left\{u_{n}\right\}_{n \in \mathrm{N}} \rightarrow u$ whenever $\left\{u_{n}\right\}_{n \in \mathrm{N}} \subset \partial K$ and

$$
\lim _{n \rightarrow \infty} \operatorname{dist}\left[\frac{u_{n}+u}{2}, \partial K\right]=0 .
$$

As mentioned in the introduction, the notion of local uniform rotundity is one of the main topics in renorming theory [6], [11], [12]. It has been traditionally considered only as a matter for norms and, consequently, closed balls. However, the definition can be easily generalized for Minkowski's gauges, as done in [7], and arbitrary convex sets, as done in the above definition.

Lemma 3.9. Let $u$ be a LUR point of the boundary of $K \in \Xi(X)$. Then, $\left\{u_{n}\right\}_{n \in \mathrm{N}} \rightarrow u$ whenever $u_{n} \in V_{K}\left(x_{n}\right)$ with $\left\{x_{n}\right\}_{n \in \mathrm{N}} \rightarrow u$.

Proof. Given the definition of the map $V_{K}$, the sequence $\left\{x_{n}\right\}_{n \in \mathrm{N}}$ is necessarily in the exterior of $K$. For each $n \in \mathrm{N}$, consider the midpoints

$$
z_{n}=\frac{u_{n}+u}{2} \quad \text { and } \quad w_{n}=\frac{u_{n}+x_{n}}{2}
$$

of the line segments $\left[u_{n}, u\right]$ and $\left[u_{n}, x_{n}\right]$, respectively (cf. Figure 2).

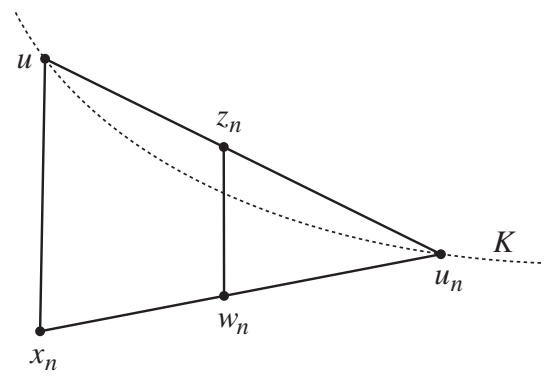

FIGURE 2. $z_{n}$ and $w_{n}$ are midpoints of $\left[u_{n}, u\right]$ and $\left[u_{n}, x_{n}\right]$, respectively.

Note that $w_{n} \notin K$ because $u_{n}$ is visible from $x_{n}$. One has

$$
\operatorname{dist}\left[z_{n}, \partial K\right] \leq\left\|z_{n}-w_{n}\right\|=(1 / 2)\left\|x_{n}-u\right\|,
$$

where the equality is due to the Thales Theorem of Proportional Line Segments (cf. [15]). Hence, $\operatorname{dist}\left[z_{n}, \partial K\right] \rightarrow 0$. Since $u$ is assumed to be LUR, the sequence $\left\{u_{n}\right\}_{n \in \mathrm{N}}$ must converge to $u$.

THEOREM 3.10. For a point $u$ in the boundary of $K \in \Xi(X)$, the following statements are equivalent:

(a) $u$ is LUR. 
(b) $\operatorname{diam}\left[V_{K}(x)\right] \rightarrow 0$ as $x$ approaches $u$ from the exterior of $K$.

Proof. Consider first the implication (a) $\Rightarrow$ (b). Take any sequence $\left\{x_{n}\right\}_{n \in \mathrm{N}}$ in $\operatorname{ext}(K)$ converging to the LUR point $u \in \partial K$. For each $n \in \mathrm{N}$, pick a pair $u_{n}, v_{n}$ of points in $V_{K}\left(x_{n}\right)$ such that

$$
\left\|u_{n}-v_{n}\right\| \geq \operatorname{diam}\left[V_{K}\left(x_{n}\right)\right]-(1 / n) .
$$

The triangle inequality yields

$$
\operatorname{diam}\left[V_{K}\left(x_{n}\right)\right] \leq(1 / n)+\left\|u_{n}-u\right\|+\left\|v_{n}-u\right\| .
$$

In view of Lemma 3.9, the last two terms in (14) go to 0. Hence, $\operatorname{diam}\left[V_{K}\left(x_{n}\right)\right]$ $\rightarrow 0$. Consider now the reverse implication $(b) \Rightarrow(a)$. Ab absurdo, suppose that $u \in \partial K$ is not LUR. In such a case, there are a positive number $r$ and sequence $\left\{u_{n}\right\}_{n \in \mathrm{N}}$ in the boundary of $K$ such that

$$
\begin{gathered}
\operatorname{dist}\left[z_{n}, \partial K\right]<1 / n, \\
\left\|u_{n}-u\right\|>r,
\end{gathered}
$$

with $z_{n}$ standing for the midpoint of the line segment $\left[u_{n}, u\right]$.

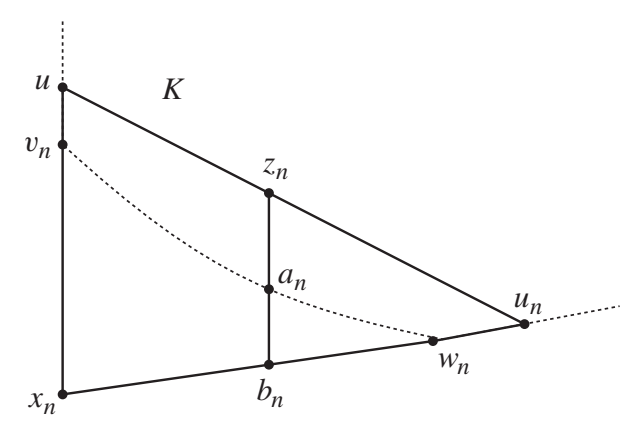

FIGURE 3. $z_{n}$ is the midpoint of $\left[u_{n}, u\right]$. The points $v_{n}$ and $w_{n}$ are visible from $x_{n}$.

We shall construct a sequence $\left\{x_{n}\right\}_{n \in \mathrm{N}}$ in the exterior of $K$ converging to $u$, but such that $\operatorname{diam}\left[V_{K}\left(x_{n}\right)\right]$ remains away from 0 . The existence of such $\left\{x_{n}\right\}_{n \in \mathrm{N}}$ would contradict the hypothesis (b). For each $n \in \mathbf{N}$, choose any $a_{n} \in \partial K$ such that $\left\|z_{n}-a_{n}\right\|<1 / n$, and then define

$$
\begin{aligned}
& b_{n}=z_{n}+2\left(a_{n}-z_{n}\right), \\
& x_{n}=u+4\left(a_{n}-z_{n}\right) .
\end{aligned}
$$

Next, let $v_{n}$ (respectively, $w_{n}$ ) be the first point that one encounters in $K$ while moving in the line segment from $x_{n}$ to $u$ (respectively, to $u_{n}$ ). It is helpful to 
have a look at Figure 3 to better understand the proof. By construction, the points $v_{n}$ and $w_{n}$ are visible from $x_{n}$, that is to say, $v_{n}, w_{n} \in V_{K}\left(x_{n}\right)$. We claim that $\left\{\left\|v_{n}-w_{n}\right\|\right\}_{n \in \mathrm{N}}$ remains away from 0 . To see this, write

$$
\begin{aligned}
\left\|v_{n}-w_{n}\right\| & \geq\left\|w_{n}-x_{n}\right\|-\left\|v_{n}-x_{n}\right\| \\
& \geq\left\|w_{n}-x_{n}\right\|-\left\|x_{n}-u\right\| \\
& \geq\left\|b_{n}-x_{n}\right\|-\left\|x_{n}-u\right\| .
\end{aligned}
$$

The term $\left\|x_{n}-u\right\|=4\left\|a_{n}-z_{n}\right\|$ goes to 0 . By contrast, the sequence $\left\{\| b_{n}-\right.$ $\left.x_{n} \|\right\}_{n \in \mathrm{N}}$ remains away from 0 , because $\left\|b_{n}-x_{n}\right\|$ is proportional to $\left\|u_{n}-u\right\|$. This proves our claim and completes the proof of the theorem.

Remark 3.11. Rolewicz [17] proved a seminal result in this direction: a Banach space $X$ is uniformly convex if and only if its closed unit ball $B_{X}$ satisfies the condition

$$
\operatorname{diam}\left[D_{B_{X}}(x) \backslash B_{X}\right] \leq \varphi(\|x\|-1)
$$

for all $x \in \operatorname{ext}\left(B_{X}\right)$, where $\varphi$ is some increasing and positive function such that $\lim _{r \rightarrow 0^{+}} \varphi(r)=0$. This result of Rolewicz can be considered as an ancestor of Theorem 3.10.

\subsection{Diameter of the visible set}

A prominent role in Theorem 3.10, and also in the sequel, is played by the function

$$
x \in \operatorname{ext}(K) \mapsto \delta_{K}(x)=\operatorname{diam}\left[V_{K}(x)\right] .
$$

Since $K$ is not necessarily bounded, the diameter of a visible set $V_{K}(x)$ may perfectly well be infinite. So, one must see (15) as an extended real valued function.

Proposition 3.12. Let $K$ be a solid closed convex set in the Euclidean space $\mathrm{R}^{d}$. Consider the following conditions:

(a) $K$ is polyhedral.

(b) $\delta_{K}$ takes a finite number of values when its argument ranges over $\operatorname{ext}(K)$.

(c) None of the boundary points of $K$ is LUR.

One has (a) $\Rightarrow(\mathrm{b})$ and $(\mathrm{b}) \Rightarrow(\mathrm{c})$, but the reverse implications are false.

Proof. We divide the proof in four parts:

(a) $\Rightarrow$ (b). This implication is a consequence of Proposition 3.7 and the fact that a polyhedral convex set has finitely many facets. One can be much more 
precise when $K$ is given by a minimal representation with a known number of facets, say $m$. The possible values taken by $\delta_{K}$ are diam $\left[\bigcup_{i \in I} F_{i}\right]$ with $I$ ranging over the collection of all nonempty subsets of $\{1, \ldots, m\}$. Hence, $\delta_{K}$ takes at most $2^{m}-1$ different values. This is only a rough upper bound. This bound can be drastically reduced, specially if one or more facets are unbounded.

(b) $\Rightarrow$ (c). Suppose, on the contrary, that $K$ admits a LUR point $u \in \partial K$. Take a sequence $\left\{x_{n}\right\}_{n \in \mathrm{N}}$ in the exterior of $K$ converging to $u$. Since $V_{K}\left(x_{n}\right)$ is neither empty, nor a singleton, one knows that $\delta_{K}\left(x_{n}\right)>0$. But $\delta_{K}\left(x_{n}\right) \rightarrow 0$ by Theorem 3.10. The conclusion is that $\delta_{K}$ takes infinitely many values on $\operatorname{ext}(K)$, contradicting the assumption (b). This proves that (b) $\Rightarrow$ (c). By the way, this implication applies in an arbitrary normed space $X$.

(b) $\nRightarrow$ (a). We construct a counterexample in $\mathrm{R}^{3}$. Let $K$ be the smallest convex set containing the point $(0,0, \sqrt{3})$ and the circle $\left\{z \in \mathrm{R}^{3}: z_{1}^{2}+z_{2}^{2} \leq\right.$ $\left.1, z_{3}=0\right\}$. The set $K$ is not polyhedral, however the function $\delta_{K}$ takes finitely many values on $\operatorname{ext}(K)$.

(c) $\nRightarrow$ (b). We construct a counterexample in $\mathrm{R}^{2}$. Let $K$ be the smallest convex set containing the points $u_{0}=(0,0)$ and $u_{1}=(0,1)$, as well as all the points of the form $u_{n}=\left(2^{-n}, 2^{-2 n}\right)$ with $n \in\{2,3, \ldots$,$\} . The boundary$ of $K$ is formed with infinitely many line segments and does not contain LUR points. However, $\delta_{K}(x)$ takes infinitely many values when $x$ moves from the exterior point $(-1,0)$ to the boundary point $(0,0)$.

The next result is a complement to Proposition 3.12. We mention it just as a mathematical curiosity because it concerns only the case of convex sets in the plane.

Proposition 3.13. Let $K$ be a solid closed convex set in the Euclidean space $\mathrm{R}^{2}$. If $K$ is nonpolyhedral, then $\delta_{K}$ takes infinitely many values when its argument ranges over $\operatorname{ext}(K)$.

Proof. Since $K \in \Xi\left(\mathrm{R}^{2}\right)$ is nonpolyhedral, it admits infinitely many extreme points. An extreme point, say $\bar{u} \in \partial K$, may be flat or not. Flatness means that, for some $\varepsilon>0$, the chord

$$
B(\bar{u}, \varepsilon) \cap \partial K=\{u \in \partial K:\|u-\bar{u}\| \leq \varepsilon\}
$$

around $\bar{u}$ is a union of two line segments. Thus, the negation of flatness indicates that $\partial K$ exhibits a certain degree of curvature around $\bar{u}$. Our proof distinguishes between two cases:

Case I: $K$ is bounded. The combination of nonpolyhedrality and boundedness ensures the existence of an extreme point $\bar{u} \in \partial K$ that is not flat, i.e., for 
all $\varepsilon>0$, the chord (16) is not a union of two line segments. Now, if one takes $\varepsilon$ small enough, then

$$
S(\bar{u}, \varepsilon) \cap \partial K=\{u \in \partial K:\|u-\bar{u}\|=\varepsilon\}
$$

contains exactly two points, say $v_{\varepsilon}$ and $w_{\epsilon}$. Not only that, these points admit supporting lines which intersect at a certain $x_{\varepsilon}$ located in ext $(K)$. What justifies the latter statement is the convexity of $K$, together with the fact that the chord (16) cannot contain both line segments $\left[\bar{u}, v_{\epsilon}\right]$ and $\left[\bar{u}, w_{\epsilon}\right]$ at the same time. Note that $V_{K}\left(x_{\varepsilon}\right)$ is contained in (16), and therefore

$$
0<\delta_{K}\left(x_{\varepsilon}\right) \leq \operatorname{diam}[B(\bar{u}, \varepsilon) \cap \partial K] \leq 2 \varepsilon .
$$

By letting $\varepsilon \rightarrow 0$, one sees that $\delta_{K}$ takes infinitely many values on $\operatorname{ext}(K)$.

Case II: $K$ is unbounded. If $K$ has an extreme point that is not flat, then we can proceed as in the previous case. Suppose then that every extreme point of $K$ is flat. The combination of nonpolyhedrality and flatness implies that the set of extreme points of $K$ has the same cardinality as N. Pick any extreme point $u_{0} \in \partial K$. Such point cut the boundary of $K$ into two branches, say $\Gamma_{1}$ and $\Gamma_{2}$. In at least one of these branches there are infinitely many extreme points. We label them $\left\{u_{n}\right\}_{n=1}^{\infty}$ in a clockwise order if they are located in $\Gamma_{1}$, or in a counter-clockwise order if they are located in $\Gamma_{2}$. To fix the ideas, suppose that we work with the branch $\Gamma_{1}$, in which case

$$
\Gamma_{1}=\bigcup_{n=0}^{\infty}\left[u_{n}, u_{n+1}\right] .
$$

For each $n \geq 0$, let $L_{n}$ be the line passing through the extreme points $u_{n}$ and $u_{n+1}$. In our specific context, a simple convexity argument (cf. Figure 4 ) shows that, for $n \geq 1$, the line $L_{n}$ meets $L_{0}$ at a unique point, which we denote by $x_{n}$.

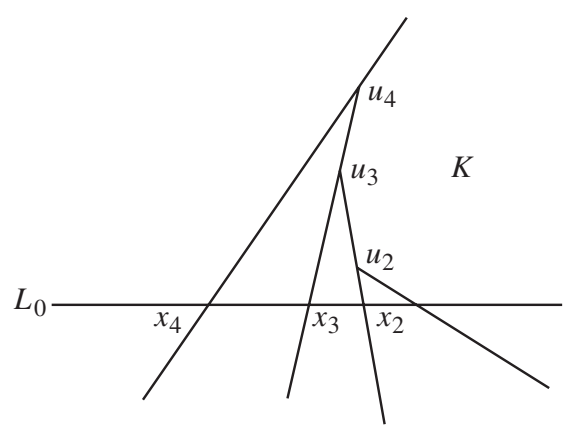

FIGURE 4. The visible sets $V_{K}\left(x_{n}\right)$ have different diameters. 
One has $x_{1}=u_{1}$, but the points $\left\{x_{n}\right\}_{n \geq 2}$ are in $\operatorname{ext}(K)$, and they move further and further away from $u_{1}$. From the very construction of $\left\{x_{n}\right\}_{n \geq 2}$, one has

$$
V_{K}\left(x_{n}\right)=\bigcup_{k=1}^{n-1}\left[u_{k}, u_{k+1}\right] .
$$

Observe also that $\delta_{K}\left(x_{n}\right)=\left\|u_{n}-u_{1}\right\|$. By letting $n \rightarrow \infty$, one sees that $\delta_{K}$ takes infinitely many values on $\operatorname{ext}(K)$.

Theorem 3.10 says that $\delta_{K}$ can be extended in a continuous manner to the LUR points in $\partial K$. It is enough to set $\delta_{K}(u)=0$ whenever $u \in \partial K$ is LUR. There is no sense in speaking of local uniform rotundity for points that are in the exterior of $K$. However, it is reasonable trying to identify the points of continuity of (15). The proposition below shows that lower-semicontinuity is not a problem at all.

Proposition 3.14. If $K \in \Xi(X)$, then $\delta_{K}$ is lower-semicontinuous as function on $\operatorname{ext}(K)$.

Proof. We see $\delta_{K}$ as the optimal-value function

$$
x \mapsto \delta_{K}(x)=\sup _{u, v \in V_{K}(x)}\|u-v\|
$$

of a parametric optimization problem. As seen in Corollary 3.2, the set $V_{K}(x)$ varies in a lower-semicontinuous manner with respect to the parameter $x$. The objective function $(u, v) \mapsto\|u-v\|$ is continuous and independent of the parameter $x$. By applying Berge's maximum theorem [1], one arrives then at the desired conclusion.

Upper semicontinuity is a more interesting business. For handling this an other issues, it is helpful to introduce a special class of points in the exterior of $K$.

Definition 3.15. A mirador of $K \in \Xi(X)$ is a point $\bar{x} \in \operatorname{ext}(K)$ at which $V_{K}: X \rightrightarrows X$ is outer-semicontinuous. The set of all miradors of $K$ is denoted by $\operatorname{mir}(K)$.

Since $V_{K}$ is lower-semicontinuous (cf. Corollary 3.2), a mirador of $K$ is a point in $\operatorname{ext}(K)$ at which $V_{K}$ behaves continuously, i.e.,

$$
\operatorname{mir}(K)=\left\{\bar{x} \in \operatorname{ext}(K): \lim _{x \rightarrow \bar{x}} V_{K}(x)=V_{K}(\bar{x})\right\} .
$$

The next proposition takes place in a finite dimensional context. It is not clear how to extend the part (a) to a more general framework. 
Proposition 3.16. Let $K$ be a bounded solid closed convex set in the Euclidean space $\mathrm{R}^{d}$.

(a) $\delta_{K}$ is continuous at each mirador of $K$.

(b) However, a point of continuity of $\delta_{K}$ is not necessarily a mirador of $K$.

Proof. In order to prove (a), one applies the upper-semicontinuous version of Berge's maximum theorem [1]. Finite dimensionality and boundedness of $K$ are used to ensure that $V_{K}$ is uniformly relatively compact, i.e., all the values of $V_{K}$ are contained in a common compact set. For showing the part (b), think of $K$ as being an equilateral triangle in the plane $\mathrm{R}^{2}$. In such a case, $\delta_{K}$ is constant on $\operatorname{ext}(K)$. Hence, it is continuous even at points which are not in $\operatorname{mir}(K)$.

The counterexample given in the above proof is not specific to the plane. In a three dimensional Euclidean space, think of $K$ as being a regular tetrahedron. Recall that a tetrahedron is a polytope composed of four triangular faces, three of which meet at each vertex. A regular tetrahedron is one in which the four triangles are equilateral. One can easily check that, when $K$ is a regular tetrahedron, the function $\delta_{K}$ is constant on $\operatorname{ext}(K)$. As explained in the next theorem, regular tethrahedra fall within a larger class of polytopes for which the function $\delta_{K}$ is constant.

THeOREm 3.17. Let $K$ be a full dimensional polytope in the Euclidean space $\mathrm{R}^{d}$. Consider the following conditions:

(a) The vertices of $K$ are pairwise equidistant.

(b) $K$ is diametrically stable, that is, each facet of $K$ has the same diameter as $K$ itself.

(c) $\delta_{K}$ is constant on $\operatorname{ext}(K)$.

(d) $\delta_{K}$ is continuous on $\operatorname{ext}(K)$.

Then, $(\mathrm{a}) \Rightarrow(\mathrm{b}) \Rightarrow(\mathrm{c}) \Leftrightarrow(\mathrm{d})$.

Proof. We divide the proof in three parts:

(a) $\Rightarrow$ (b). Express the polytope $K$ as convex envelope

$$
K=\operatorname{co}\left\{w_{0}, w_{1}, \ldots, w_{p}\right\}
$$

of its vertices. Denote by $c$ the common distance between any pair of vertices. Since a polytope admits always a pair of vertices attaining its diameter, one has

$$
\operatorname{diam}(K)=\max _{0 \leq i, j \leq p}\left\|w_{i}-w_{j}\right\|=c .
$$


On the other hand, any facet of (17) is expressible in the form $F=\operatorname{co}\left\{w_{i}\right.$ : $i \in I\}$ with $I$ containing at least two indices taken from $\{0,1, \ldots, p\}$. Hence,

$$
\operatorname{diam}(F)=\max _{i, j \in I}\left\|w_{i}-w_{j}\right\|=c .
$$

This shows that $K$ is diametrically stable.

(b) $\Rightarrow$ (c). The proof of this implication is based on Proposition 3.7. Take any $x \in \operatorname{ext}(K)$. The visible set $V_{K}(x)$ is expressible as union of facets of $K$, say $V_{K}(x)=F_{1} \cup \ldots \cup F_{r}$. Hence,

$$
\operatorname{diam}(K)=\operatorname{diam}\left(F_{1}\right) \leq \operatorname{diam}\left[V_{K}(x)\right] \leq \operatorname{diam}(K) .
$$

This proves that $\delta_{K}(x)=\operatorname{diam}(K)$ for all $x \in \operatorname{ext}(K)$.

(c) $\Leftrightarrow$ (d). In view of Proposition 3.12, the function $\delta_{K}$ takes finitely many values on $\operatorname{ext}(K)$. Hence, continuity is equivalent to constancy.

Concerning Theorem 3.17, an interesting open question is whether the constancy of $\delta_{K}$ implies that $K$ is diametrically stable. The answer is yes, for instance, if one knows that $K$ has exactly $d+1$ vertices. Unfortunately, we do not have a clear answer in the general case. The following result is specific to a two dimensional setting.

Proposition 3.18. Let $K$ be a compact solid convex set in $\mathrm{R}^{2}$. If $\delta_{K}$ is constant on $\operatorname{ext}(K)$, then $K$ is an equilateral triangle.

Proof. Suppose that $\delta_{K}(x)=c$ for all $x \in \operatorname{ext}(K)$. By Proposition 3.13, $K$ must be polyhedral. Hence, one can represent this set as in (17). Since $K$ is full dimensional in $\mathrm{R}^{2}$, one has $p \geq 2$. The vertices can be labeled in a clockwise order, so that $\left[w_{0}, w_{1}\right], \ldots,\left[w_{p-1}, w_{p}\right],\left[w_{p}, w_{0}\right]$ are the sides of $K$. The constancy assumption made on $\delta_{K}$ implies that all the sides of $K$ have $c$ as common length. Hence, $K$ is a $(p+1)$-sided regular polygone with angle between two consecutive sides given by

$$
\theta=\left(1-\frac{2}{p+1}\right) \pi \geq \frac{\pi}{3}
$$

On the other hand, there is some $x \in \operatorname{ext}(K)$ such that $V_{K}(x)=\left[w_{0}, w_{1}\right] \cup$ $\left[w_{1}, w_{2}\right]$. Since the diameter of this union is also equal to $c$, it follows that $\theta \leq \pi / 3$. In view of (18), one gets $\theta=\pi / 3$ and $p=2$. This proves that $K$ is an equilateral triangle.

REMARK 3.19. In a three dimensional Euclidean space, consider the tethra- 
hedron $K$ with vertices

$$
\begin{aligned}
& w_{0}=(0,0,0) \\
& w_{1}=(2,0,0) \\
& w_{2}=(1, \sqrt{3}, 0) \\
& w_{3}=(1, \sqrt{75 / 36}, \sqrt{11 / 12}) .
\end{aligned}
$$

All the edges of $K$ have length equal to 2, except for the edge $\left[w_{2}, w_{3}\right]$ which has unit length. Note that $K$ is diametrically stable. Although $\delta_{K}$ is constant on $\operatorname{ext}(K)$, the tethahedron $K$ is not regular.

\section{From visibility to weak visibility}

We end this work by comparing visibility and weak visibility. Recall that visible sets may not be closed. In contrast, weakly visible sets are always closed. Not only that, the latter sets behave outer-semicontinuously.

Proposition 4.1. Let $K \in \Xi(X)$. Then, $W_{K}: X \rightrightarrows X$ is outer-semicontinuous at each $\bar{x} \in \operatorname{ext}(K)$, and

$$
W_{K}(\bar{x})=\limsup _{x \rightarrow \bar{x}} V_{K}(x)=\limsup _{x \rightarrow \bar{x}} L_{K}(x) .
$$

Proof. Let $\bar{x} \in \operatorname{ext}(K)$. Take convergent sequences $\left\{x_{n}\right\}_{n \in \mathrm{N}} \rightarrow \bar{x}$ and $\left\{u_{n}\right\}_{n \in \mathrm{N}} \rightarrow \bar{u}$ such that $u_{n} \in W_{K}\left(x_{n}\right)$ for all $n \in \mathrm{N}$. The limit $\bar{u}$ is necessarily in $\partial K$. We must prove that

$$
[\bar{x}, \bar{u}] \cap \operatorname{int}(K)=\emptyset .
$$

Suppose, on the contrary, that the line segment $[\bar{x}, \bar{u}]$ meets the open set int $(K)$. In such a case, $[x, u] \cap \operatorname{int}(K) \neq \emptyset$ for all $(x, u)$ in some neighborhood of $(\bar{x}, \bar{u})$, a contradiction with the fact that $u_{n} \in W_{K}\left(x_{n}\right)$ for all $n \in \mathrm{N}$. This shows that $W_{K}$ is outer-semicontinuous at $\bar{x}$. The second equality in (19) is a consequence of (2) and Proposition 3.1(b). The inclusion

$$
\limsup _{x \rightarrow \bar{x}} V_{K}(x) \subset W_{K}(\bar{x}) .
$$

is a consequence of (2) and the outer-semicontinuity of $W_{K}$. For proving the reverse of (21), take $\bar{u} \in W_{K}(\bar{x})$. If $\bar{u} \in V_{K}(\bar{x})$, then we are done. If $\bar{u} \notin V_{K}(\bar{x})$, then there exists a point $w \in \partial K$ different from $\bar{u}$ such that $w \in[\bar{x}, \bar{u}]$. Since $\bar{u}$ is weakly visible from $\bar{x}$, we know that $\bar{u} \in \partial K$ and that the relation (20) holds. A nonstrict separation argument guarantees the existence of a nonzero linear continuous function $f: X \rightarrow \mathrm{R}$ and a scalar $\beta \in \mathrm{R}$ such that $f(z) \leq \beta$ for all 
$z \in K$, and $f(y) \geq \beta$ for all $y \in[\bar{x}, \bar{u}]$. Since $\bar{u}$ and $w$ are in $[\bar{x}, \bar{u}] \cap \partial K$, one gets $f(\bar{u})=f(w)=\beta$. It follows that $f(\bar{x})=\beta$, because $w$ is a convex combination of $\bar{x}$ and $\bar{u}$. In such a case, one can construct a sequence $\left\{x_{n}\right\}_{n \in \mathrm{N}} \rightarrow \bar{x}$ with $f\left(x_{n}\right)>\beta$ for all $n \in \mathrm{N}$. Note that the line segment $\left[x_{n}, \bar{u}\right]$ intersects $K$ only at $\bar{u}$, i.e., $\bar{u}$ is visible from $x_{n}$. Hence, $\bar{u} \in \lim _{\sup _{x \rightarrow \bar{x}}} V_{K}(x)$, and the proof is complete.

REMARK 4.2. We have shown something slightly stronger than the first equality in (19), namely, $W_{K}^{-1}(\bar{u})=\mathrm{cl}\left[V_{K}^{-1}(\bar{u})\right]$ for all $\bar{u}$ in the boundary of $K$.

Miradors are not too difficult to identify after all. Necessary and sufficient conditions for a point to be a mirador are given in the next theorem.

Theorem 4.3. Let $K \in \Xi(X)$ and $\bar{x} \in \operatorname{ext}(K)$. Then, the following conditions are equivalent:

(a) Any line passing through $\bar{x}$ meets $\partial K$ at most in two points.

(b) $V_{K}(\bar{x})=W_{K}(\bar{x})$.

(c) $\bar{x}$ is a mirador of $K$.

Proof. That $W_{K}(\bar{x}) \neq V_{K}(\bar{x})$ is equivalent to the existence of a point that is weakly visible from $\bar{x}$, but not visible from $\bar{x}$. In turn, this is equivalent to the existence of two different points $u, w \in \partial K$ such that $w \in[\bar{x}, u]$ and $[w, u] \subset \partial K$. This proves that (a) $\Leftrightarrow(\mathrm{b})$. Proposition 4.1 takes care of the equivalence between (b) and (c).

Theorem 4.3 has several interesting consequences. The corollary stated below is just an example.

Corollary 4.4. Let $K \in \Xi(X)$. Then, the following conditions are equivalent:

(a) $K$ is strictly convex in the sense that $\partial K$ does not contain a line segment.

(b) $V_{K}(x)=W_{K}(x)$ for all $x \in \operatorname{ext}(K)$.

Proof. It is immediate from the equivalence (a) $\Leftrightarrow$ (b) in Theorem 4.3.

\section{Conclusions}

The conclusions drawn here concern not just the present work, but also the companion work [14]. The strategy adopted in both papers has been examining the geometric nature of a convex set $K$, and specially of its boundary $\partial K$, by relying on various sets

$$
T_{K}(x), D_{K}(x), L_{K}(x), V_{K}(x), W_{K}(x), \ldots
$$


that depend on a certain parameter $x \in X$. As a general rule, $x$ moves in the exterior of $K$.

Sometimes we let $x$ approach the boundary and, on other occasions, we allow $x$ to stay away from the boundary. In any case, the semicontinuity behavior of the multivalued maps $T_{K}, D_{K}, L_{K}, V_{K}$, and $W_{K}$, provides a good insight of the geometry of the set $K$ itself. Table 1 summarizes the situation, as far as lower- and outer-semicontinuity are concerned.

\begin{tabular}{|c|c|c|}
\hline \multirow{2}{*}{ Map } & \multicolumn{2}{|c|}{ Type of semicontinuity } \\
\cline { 2 - 3 } & Lower & Outer \\
\hline$T_{K}$ & yes & yes \\
\hline$D_{K}$ & yes & yes \\
\hline$L_{K}$ & yes & no \\
\hline$V_{K}$ & yes & no \\
\hline$W_{K}$ & no & yes \\
\hline
\end{tabular}

TABLE 1 . Semicontinuity behavior at points in the exterior of $K$.

We have obtained several results which fit into a common mould, for instance,

$\partial K$ is segment free $\Longleftrightarrow V_{K}$ is outer-semicontinous on $\operatorname{ext}(K)$,

$\partial K$ is line free $\Longleftrightarrow T_{K}$ is injective on $\operatorname{ext}(K)$,

and so on. As prototype of result that concentrates on a specific boundary point $u \in \partial K$, one has

$$
\begin{aligned}
u \text { is LUR } & \Longleftrightarrow \operatorname{diam}\left[V_{K}(x)\right] \rightarrow 0 \text { as } x \rightarrow u, \\
u \text { is smooth } & \Longleftrightarrow \Phi_{\text {frob }}\left[T_{K}(x)\right] \rightarrow 1 \text { as } x \rightarrow u \text { with } x \gg_{K} u .
\end{aligned}
$$

The details of these statements can be consulted in the proper place.

There are probably many other results that can be established along the same lines. The external analysis of convex sets is a field that still offers a wide range of possibilities. General questions concerning the geometry of convex sets could be handled with such an approach.

\section{REFERENCES}

1. Berge, C., Espaces Topologiques, Fonctions Multivoques, Dunod, Paris 1966.

2. Bezdek, K., On the illumination of smooth convex bodies, Arch. Math. (Basel) 58 (1992), 611-614. 
3. Bezdek, K., The illumination conjecture and its extensions, Period. Math. Hungar. 53 (2006), 59-69.

4. Bogopolskii, O. V., and Vasiliev, V. A., On the multiplicity of the illumination of convex bodies by point sources, Math. Notes 54 (1993), 987-991.

5. Buchman, E., and Valentine, F. A., External visibility, Pacific J. Math. 64 (1976), 333-340.

6. Deville, R., Godefroy, G., and Zizler, V., Smoothness and Renormings in Banach Spaces, Pitman Monographs 64, Wiley, New York 1993.

7. Georgiev, P. G., Granero, A. S., Jiménez Sevilla, M., and Moreno, J. P., Mazur intersection properties and differentialiblity of convex functions in Banach spaces, J. London Math. Soc. (2) 61 (2000), 531-542.

8. Hadwiger, H., Ungelöste Probleme, Nr. 38, Elem. Math. 15 (1960), 130-131.

9. Kutzarova, D. N., and Rolewicz, S., On drop property for convex sets, Arch. Math. (Basel) 56 (1991), 501-511.

10. Martini, H., and Soltan, V., Combinatorial problems on the illumination of convex bodies, Aequationes Math. 57 (1999), 121-152.

11. Moltó, A., Orihuela, J., Troyanski, S., and Valdivia, M., On weakly locally uniformly rotund Banach spaces, J. Funct. Anal. 163 (1999), 252-271.

12. Moltó, A., Orihuela, J., Troyanski, S., and Valdivia, M., A Nonlinear Transfer Technique for Renorming, Lecture Notes in Math., Springer, Berlin 2009.

13. Montesinos, V., Drop property equals reflexivity, Studia Math. 87 (1987), 93-100.

14. Moreno, J. P., and Seeger, A., External analysis of boundary points of convex sets: supporting cones and drops, J. Convex Analysis 16 (2009), in press.

15. Patsopoulos, D., and Patronis, T., The theorem of Thales: a study of the naming of theorems in school geometry textbooks, Int. J. Hist. Math. Educ. 1 (2006), 57-68.

16. Rockafellar, R. T., and Wets, R. J.-B., Variational Analysis, Grundlehren der math. Wiss. 317, Springer, Berlin 1998.

17. Rolewicz, S., On drop property, Studia Math. 85 (1986), 27-35.

18. Schneider, R., Convex Bodies: The Brunn-Minkowski Theory, Encyclopedia Math. Appl. 44, Cambridge Univ. Press, Cambridge 1993.

19. Valentine, F. A., Visible shorelines, Amer. Math. Monthly 77 (1970), 146-152.

DEPARTAMENTO DE MATEMÁTICAS

FACULTAD DE CIENCIAS

UNIVERSIDAD AUTÓNOMA DE MADRID

MADRID 28049

SPAIN

E-mail: josepedro.moreno@uam.es
DEPARTMENT OF MATHEMATICS

UNIVERSITY OF AVIGNON

33 RUE LOUIS PASTEUR

84000 AVIGNON

FRANCE

E-mail: alberto.seeger@univ-avignon.fr 